Portland State University

PDXScholar

$12-1-1980$

\title{
Stopping power of matter for alpha particles at extreme relativistic energies
}

\author{
P.T. Leung \\ Portland State University \\ M. L. Rustgi \\ J. E. Turner
}

Follow this and additional works at: https://pdxscholar.library.pdx.edu/phy_fac

Part of the Physics Commons

Let us know how access to this document benefits you.

\section{Citation Details}

Rustgi, M. L., Turner, J. E., \& Leung, P. T. (1980). Stopping power of matter for alpha particles at extreme relativistic energies. Physical Review A (General Physics), 22(6), 2510-2512.

This Article is brought to you for free and open access. It has been accepted for inclusion in Physics Faculty Publications and Presentations by an authorized administrator of PDXScholar. Please contact us if we can make this document more accessible: pdxscholar@pdx.edu. 


\title{
Stopping power of matter for alpha particles at extreme relativistic energies
}

\author{
M. L. Rustgi and J. E. Turner \\ Health and Safety Research Division, Oak Ridge National Laboratory, Oak Ridge, Tennessee 37830 \\ P. T. Leung \\ Physics Department, State University of New York, Buffalo, New York 14260
}

(Received 21 July 1980)

\begin{abstract}
The stopping power of matter for alpha particles at extreme relativistic energies has been calculated by incorporating the charge form factor. A table is presented for aluminum, copper, and lead. It is found that at the highest energies considered here, inclusion of form factor reduces the mass stopping power by about 6-8\% in comparison with that predicted by Bethe's relativistic formula.
\end{abstract}

The stopping power of matter for heavy charged particles (muon, proton, etc.) is described by Bethe's relativistic formula ${ }^{1,2}$

$$
-d E / d s=\kappa\left[\ln \left(2 \gamma^{2} m v^{2} / I\right)-\beta^{2}\right] .
$$

Here $\kappa=4 \pi z^{2} e^{4} N Z / m v^{2}$, where $z e$ and $v$ are the charge and speed of the incident particle, $-e$ is the electronic charge, $N Z$ represents the number of electrons per unit volume in the medium, $\beta$ $=v / c$ is the speed of the particle relative to the speed of light, $\gamma^{2}=1 /\left(1-\beta^{2}\right)$, and $I$ is the mean excitation energy of the medium. In deriving Eq. (1), the theory is simplified by assuming that

$$
\gamma m / M \ll 1,
$$

where $M$ is the rest mass of the incident particle. In this case the heavy particle can lose only a small fraction of its energy in a single atomic collision. The dependence of the stopping power on speed is then the same for all particles, as expressed by Eq. (1). Condition (2) breaks down at very high energies when $\gamma$ becomes large. The stopping power then depends on other factors such as $M$, the particle's spin and internal structure. The last property can be expressed by means of the form factors for the distribution of charge and magnetic moment. At extreme relativistic energies the stopping-power formula depends on the particular particle under consideration. In this paper we calculate the stopping power for energetic alpha particles without employing restriction (2). Earlier companion papers have treated the muon and proton ${ }^{3}$ and the deuteron. ${ }^{4}$

The differential cross section for the scattering of an electron at an angle $\theta$ from an $\alpha$ particle at rest may be written as

$$
\frac{d \sigma}{d \Omega}=\left(\frac{z e^{2}}{2 \gamma m v^{2}}\right)^{2} \frac{\cos ^{2} \frac{1}{2} \theta}{\sin ^{4} \frac{1}{2} \theta} \frac{F_{E}^{2}\left(q^{2}\right)}{\left[1+\left(2 \gamma m / M_{\alpha}\right) \sin ^{2} \frac{1}{2} \theta\right]},
$$

where $\hbar q$ is the magnitude of the change in the electron's (= alpha particle's) energy-momentum four vector, $M_{\alpha}$ is the mass of the alpha particle, and $F_{E}$ is the charge form factor of the alpha particle. This factor is related to the bare form factor $F_{B}\left(q^{2}\right)$ through the equation

$$
F_{E}\left(q^{2}\right)=F_{B}\left(q^{2}\right) \times F_{E S}\left(q^{2}\right),
$$

where

$$
F_{E S}=F_{E p}+F_{E n}
$$

is the isoscalar form factor. The bare form factor is related to the Fourier transform of the squared $\alpha$-particle wave function, i.e. ,

$$
\begin{aligned}
F_{B}=\frac{1}{2\left(1+C^{2}\right)} \int & \left|\psi_{s}+C \psi_{D}\right|^{2} \\
\times & \left(\exp \frac{i}{2} \overrightarrow{\mathrm{q}} \cdot(\overrightarrow{\mathrm{u}}-\sqrt{2} \overrightarrow{\mathrm{w}})\right. \\
& \left.\quad+\exp \frac{i}{2} \overrightarrow{\mathrm{q}} \cdot(\overrightarrow{\mathrm{u}}+\sqrt{2} \overrightarrow{\mathrm{w}})\right) d \overrightarrow{\mathrm{u}} d \overrightarrow{\mathrm{v}} d \overrightarrow{\mathrm{w}},
\end{aligned}
$$

where

$$
\overrightarrow{\mathrm{a}}=\frac{1}{2}\left(\overrightarrow{\mathrm{r}}_{3}+\overrightarrow{\mathrm{r}}_{4}-\overrightarrow{\mathrm{r}}_{2}-\overrightarrow{\mathrm{r}}_{1}\right),
$$

and

$$
\overrightarrow{\mathrm{v}}=\left(\overrightarrow{\mathrm{r}}_{2}-\overrightarrow{\mathrm{r}}_{1}\right) / \sqrt{\mathbf{2}}, \quad \overrightarrow{\mathrm{w}}=\left(\overrightarrow{\mathrm{r}}_{4}-\overrightarrow{\mathrm{r}}_{3}\right) / \sqrt{\mathbf{2}} .
$$

Here $\vec{r}_{1}$ and $\vec{r}_{2}$ denote the neutron and $\vec{r}_{3}$ and $\overrightarrow{\mathbf{r}}_{4}$ the proton position vectors, and $\psi_{S}$ and $\psi_{D}$ are the wave functions of the admixture of the ${ }^{1} S_{0}$ and ${ }^{5} D_{0}$ states of the alpha particle.

By carrying out the integrations, it has been shown by Singh et al..$^{5}$ that

$$
F_{B}=\frac{1}{\left(1+C^{2}\right)}\left(\frac{1}{\left(1+3 q^{2} / 64 \alpha^{2}\right)^{5}}+\frac{C^{2}\left(1-\frac{19}{192} q^{2} / \beta^{2}\right)}{\left(1+3 q^{2} / 64 \beta^{2}\right)^{8}}\right),
$$


TABLE I. Values of $F_{B}^{2} F_{B S}^{2}$ for various values of $Q$.

\begin{tabular}{rlc}
\hline \hline$Q$ & Fitted & $\begin{array}{c}\text { Calculated from } \\
\text { Eqs. (9) and (10) }\end{array}$ \\
\hline 0 & 1.0 & 1.0 \\
100 & 0.1254 & 0.1254 \\
200 & $2.0787 \times 10^{-2}$ & $2.0771 \times 10^{-2}$ \\
300 & $4.2608 \times 10^{-3}$ & $4.2565 \times 10^{-3}$ \\
400 & $1.0314 \times 10^{-3}$ & $1.0314 \times 10^{-3}$ \\
500 & $2.8546 \times 10^{-4}$ & $2.8618 \times 10^{-3}$ \\
600 & $8.8203 \times 10^{-5}$ & $8.8815 \times 10^{-5}$ \\
700 & $2.9882 \times 10^{-5}$ & $3.0280 \times 10^{-5}$ \\
\hline \hline
\end{tabular}

where $\alpha=0.841 \times 10^{13} \mathrm{~cm}^{-1}, \beta=1.365 \times 10^{13} \mathrm{~cm}^{-1}$, and $C=-0.153$. According to Dudelzak ${ }^{6}$

$$
F_{E S}=\frac{2.28}{\left(1+0.06345 q^{2}\right)}-\frac{1.28}{\left(1+0.03739 q^{2}\right)} .
$$

In order to calculate the extreme relativistic contributions to this stopping-power formula, let us denote the energy lost by the alpha particle in a single collision by

$$
Q=\hbar^{2} q^{2} / 2 m \text {. }
$$

It is easily shown that the maximum energy $Q_{m}$ that can be lost is given by $\left(m / M_{\alpha} \ll 1\right)$ :

$$
Q_{m}=2 \gamma^{2} m v^{2} /\left(1+2 \gamma m / M_{\alpha}\right) \text {. }
$$

For $q \leqslant 4 F^{-1}\left(F=10^{-13} \mathrm{~cm}\right), Q_{m} \leqslant 610 \mathrm{GeV}$, and $\gamma \leqslant 10^{4}$. We now express each of the factors in Eqs. (3), (9), and (10) in terms of $Q$, the alpha particle's energy loss. It is found that

$$
d \sigma=2 \pi \frac{z^{2} e^{4}}{m v^{2}} \frac{d Q}{Q^{2}}\left(1-\frac{Q \beta^{2}}{Q_{m}}\right) \sum_{i=1}^{2} \frac{A_{i}}{\left(1+a_{i} Q\right)^{12}} .
$$

In writing (13) we have fitted $F_{B}^{2} F_{E S}^{2}$ by

$$
\sum \frac{A_{i}}{\left(1+a_{i} Q\right)^{12}}
$$

with $a_{1}=1.787 \times 10^{-3} \mathrm{GeV}^{-1}, a_{2}=1.7 \times 10^{-3} \mathrm{GeV}^{-1}$, $A_{1}=9.2099$, and $A_{2}=1-A_{1}$. The accuracy of the fit can be seen from Table I.

The contribution of distant collisions to the stopping power of a medium is given by

$$
-\left(\frac{d E}{d s}\right)_{Q<\eta}=\frac{1}{2} \kappa\left[\ln \left(2 \gamma^{2} m v^{2} \eta / I^{2}\right)-\beta^{2}\right],
$$

where $\eta$ is an intermediate value of energy loss. ${ }^{2,7}$ The contribution of close collisions is given by

$$
-\left(\frac{d E}{d s}\right)_{Q>\eta}=N Z \int_{\eta}^{Q_{m}} Q d \sigma \text {. }
$$

Multiplying both sides of (13) by $N Z Q$, integrating, and adding the result to $-(d E / d s)_{Q<\eta}$, we find for the stopping power $\left(a_{i} \eta \ll 1\right)$

$$
\begin{aligned}
-\left(\frac{d E}{d s}\right)=\kappa & {\left[\ln \frac{2 \gamma^{2} m v^{2}}{I}-\frac{\beta^{2}}{2}-\frac{1}{2} \sum_{i} A_{i} \ln \left(1+a_{i} Q_{m}\right)+\frac{1}{2} \ln \frac{M_{\alpha}}{M_{\alpha}+2 \gamma m}-\frac{1}{2}\left(\sum_{r=1}^{11} \frac{1}{r}+\frac{\beta^{2}}{11 Q_{m}} \sum_{i} \frac{A_{i}}{a_{i}}\right)\right.} \\
& \left.+\sum_{r=1}^{11} \sum_{i} \frac{A_{i}}{2 r\left(1+a_{i} Q_{m}\right)^{r}}+\sum_{i} \frac{\beta^{2}}{22 Q_{m}} \frac{A_{i}}{a_{i}} \frac{1}{\left(1+a_{i} Q_{m}\right)^{11}}\right] .
\end{aligned}
$$

This formula is valid when $\gamma \lessgtr 10^{4}$ and can be simplified if $a_{i} Q_{m} \ll 1$. In this approximation $\left(a_{i} Q_{m}\right.$ $\ll 1)$, (15) becomes

$$
\begin{aligned}
-\left(\frac{d E}{d s}\right)=\kappa( & \ln \frac{2 \gamma^{2} m v^{2}}{I}-\beta^{2} \\
& \left.+\frac{1}{2} \ln \frac{M_{\alpha}}{M_{\alpha}+2 \gamma m}-6 \sum_{i} A_{i} a_{i} Q_{m}\right) .
\end{aligned}
$$

Equations (15), (16), and (1) were used to calcu- late the mass stopping power $(-1 / \rho) d E / d s$ for $\mathrm{Al}$,

\begin{tabular}{|c|c|c|c|c|c|c|c|c|c|c|}
\hline$\gamma$ & $\begin{array}{l}\text { Alpha } \\
\text { energy } \\
(\mathrm{GeV})\end{array}$ & Eq. (15) & $\begin{array}{c}\mathrm{Al} \\
\text { Eq. (1) }\end{array}$ & Eq. (16) & Eq. (15) & $\begin{array}{c}\text { Cu } \\
\text { Eq. (1) }\end{array}$ & Eq. (16) & Eq. (15) & $\begin{array}{c}\mathrm{Pb} \\
\text { Eq. (1) }\end{array}$ & Eq. (16) \\
\hline 10 & 37.3 & 7.385 & 7.386 & 7.385 & 6.620 & 6.621 & 6.620 & 5.268 & 5.270 & 5.268 \\
\hline 50 & 186 & 9.210 & 9.222 & 9.202 & 8.353 & 8.364 & 8.344 & 6.775 & 6.785 & 6.768 \\
\hline 100 & 373 & 10.001 & 10.041 & 9.967 & 9.102 & 9.139 & 9.069 & 7.425 & 7.458 & 7.397 \\
\hline 250 & 932 & 10.945 & 11.125 & & 9.996 & 10.166 & & 8.201 & 8.348 & \\
\hline 500 & 1864 & 11.503 & 11.945 & & 10.525 & 10.944 & & 8.659 & 9.022 & \\
\hline 750 & 2796 & 11.777 & 12.426 & & 10.783 & 11.399 & & 8.883 & 9.417 & \\
\hline 1000 & 3728 & 11.959 & 12.767 & & 10.956 & 11.722 & & 9.033 & 9.697 & \\
\hline
\end{tabular}
$\mathrm{Cu}$, and $\mathrm{Pb}$. The mean ionization potentials $I$ for these elements were taken to be 163,316 , and $825 \mathrm{eV}$, respectively. The results are listed in Table II. It is seen that at higher energies $(\gamma \sim 1000)$, the form-factor effects decrease the mass stopping power by about $6-8 \%$. The above calculations were also carried out with the wave function of Jain and Srivastava ${ }^{8}$ without any significant difference in the results.

TABLE II. Mass stopping power of $\mathrm{Al}, \mathrm{Cu}$, and $\mathrm{Pb}$ for alpha particles at extreme relativistic energies. 


\section{ACKNOWLEDGMENT}

This research was sponsored in part by the Office of Health and Environmental Research, U.S.
Department of Energy, under Contract No. W-7405-ENG-26 with the Union Carbide Corporation.
${ }^{1} \mathrm{H}$. A. Bethe, in Handbuch der Physik, edited by H. Geiger and K. Scheel (Springer, Berlin, 1933), Vol. 24/1, pp. $491 \mathrm{ff}$.

${ }^{2}$ U. F ano, Annu. Rev. Nucl. Sci. 13, 1 (1963).

${ }^{3}$ J. E. Turner, V. N. Neelavathi, R. B. Vora, T. S. Subramanian, and M. A. Prasad, Phys. Rev. 183, 453 (1969).

${ }^{4}$ R. B. Vora and J. E. Turner, Phys. Rev. B 1 , 2011 (1970).

${ }^{5}$ R. P. Singh, R. Raj, and M. L. Rustgi, Nucl. Phys. A
133, 365 (1969).

6 B. Dudelzak, thesis, University of Paris, Orsay, Serie A. N. D'Ordre, 1965 (unpublished); J. S. Levinger, Phys. Rev. 162, 1589 (1967); J. S. Levinger, in Perspectives in Modern Physics, edited by R. Marshak (Wiley, New York, 1966).

${ }^{7}$ E. A. Uehling, Annu. Rev. Nucl. Sci. 4, 315 (1954).

${ }^{8}$ S. C. Jain and B. K. Srivastava, Phys. Rev. 164, 1226 (1967). 